\title{
Associative grouping: Perceptual grouping of shapes by association
}

\author{
TIMOTHY J. VICKERY \\ Yale University, New Haven, Connecticut \\ AND \\ YUHONG V. JiANG \\ University of Minnesota, Minneapolis, Minnesota
}

\begin{abstract}
Perceptual grouping is usually defined by principles that associate distinct elements by virtue of image properties, such as proximity, similarity, and occurrence within common regions. What role does learning play in forming a perceptual group? This study provides evidence that learning of shape associations leads to perceptual grouping. Subjects were repeatedly exposed to pairs of unique shapes that co-occurred within a common region. The common region cue was later removed in displays composed of these shapes, and the subjects searched the display for two adjacent shapes of the same color. The subjects were faster at locating the color repetition when the adjacent shapes with the same color came from the same trained groups than when they were composed of two shapes from different trained groups. The effects were perceptual in nature: Learned pairings produced spatial distortions similar to those observed for groups defined by perceptual similarity. A residual grouping effect was observed even when the shapes in the trained group switched their relative positions but was eliminated when each shape was inverted. These results indicate that statistical co-occurrence with explicit grouping cues may form an important component of perceptual organization, determining perceived scene structure solely on the basis of past experience.
\end{abstract}

A fundamental challenge for vision science lies in determining how humans detect and understand structure in the visual environment. Historically, the processes that create an organized percept have been seen as driven primarily by stimulus properties, such as texture continuity, similarity and proximity of distinct elements, and the presence of cues to region and connectedness in the image (Palmer, 1992; Palmer \& Rock, 1994; Wertheimer, 1923). Vision scientists have long viewed perceptual organization as a set of processes that precede object recognition and the operation of visual attention (e.g., Marr, 1982; Neisser, 1967; Treisman, 1982), and Gestalt psychologists especially viewed these processes as largely independent of experience (e.g., Gottschaldt, 1926; Wertheimer, 1923). However, some of these views have been challenged in recent years. For instance, Peterson and colleagues provided evidence that figure-ground segmentation, a fundamental component of perceptual organization, is profoundly influenced by knowledge of familiar shapes (Peterson \& Gibson, 1994; Peterson, Harvey, \& Weidenbacher, 1991). This influence of learning on perceptual organization opens up the possibility that learning may also alter the perceptual grouping of distinct visual elements, a notion taken for granted by recent theories of grouping (Roelfsema, 2006; Ullman, 2007). However, as will be reviewed below, empirical evidence for how learning affects per- ceptual grouping remains ambiguous. Here, we attempt to answer the question, Can two shapes become so strongly associated through learning that they are unintentionally grouped?

In this article, we address perceptual grouping, which must be distinguished from other perceptual organization processes, such as texture segmentation (resolution of a continuous region on the basis of textural differences) and figure-ground segmentation (assignment of depth to elements of a scene that border one another in the image). Perceptual grouping binds together distinct elements that do not necessarily share borders in an image (Palmer \& Rock, 1994). Perceptual space around the grouped items is warped (Coren \& Girgus, 1980), and attention spreads within groups or preferentially selects grouped elements (Dodd \& Pratt, 2005; Marino \& Scholl, 2005). The outcome of perceptual grouping is a representation that is, on some level, treated as a whole by attention and perception, even though it clearly consists of distinct and, often, widely separated elements.

\section{Previous Demonstrations of \\ Experience Effects in Grouping}

Recent evidence suggests that experience plays a significant role in some aspects of perceptual grouping. In the most direct demonstration to date of the importance 
of learning in grouping, Zemel, Behrmann, Mozer, and Bavelier (2002) asked subjects to compare the contour properties of object parts situated on opposite sides of an occluding bar. This comparison was faster when the parts were aligned and appeared to belong to the same object than when they were offset and seen as parts of different objects. However, some subjects were trained on the task in a session in which the occluder was removed, and the unaligned elements were seen as part of the same object. This training changed the subjects' assumptions about the occluded shapes, and the unaligned parts were perceived as belonging to the same objects when the occluder was in place; these subjects compared unaligned regions as quickly as aligned regions. Thus, learning altered the interpretation of amodal completion, by inducing observers to treat two widely separated image segments as part of a single object.

In another demonstration of the effects of experience on grouping, Kimchi and Hadad (2002) asked subjects to judge whether two side-by-side letter shapes were the same or different. These shapes were preceded by another shape that was either the same as one or both targets or unrelated (an array of random dots). Kimchi and Hadad found that a related prime sped up same judgments, both when the prime and targets were upright and when they were inverted. However, when the prime letter segments were constructed of disconnected segments, briefly presented primes facilitated later comparison only in the upright orientation for short prime-target intervals. Kimchi and Hadad suggested that past experience with upright letters enabled the subjects to quickly group segments together when they formed a letter. Lack of experience with inverted letters eliminated this grouping advantage.

Although the studies cited above have implied a role of learning in modulating perceptual grouping, we do not know whether such learning depends on the presence of amodal completion cues (as was the case in Zemel et al.'s [2002] study) and whether a short period of training (as opposed to a lifelong experience with letters, as in Kimchi and Hadad's [2002] study) can affect perceptual grouping. The focus of the present study was to examine learned grouping among disconnected elements following a short learning period.

\section{Evidence From Illusory Conjunctions}

Prinzmetal and colleagues have examined a question similar to that posed by our study, using illusory conjunctions, which occur when features (e.g., color) of one object are inappropriately bound to features (e.g., shape) of another object (Treisman \& Schmidt, 1982). Illusory conjunctions are more likely to occur within perceptual groups than between groups (Ivry \& Prinzmetal, 1991; Prinzmetal \& Keysar, 1989), and thus they have sometimes been used as an index of grouping. In an examination of the effect of experience on illusory conjunctions, Prinzmetal and colleagues found that illusory conjunctions of letter shape and color were more likely to be observed between elements of an orthographic unit than between elements of two different orthographic units (Prinzmetal \& Millis-Wright, 1984; Prinzmetal, Treiman, \& Rho, 1986).
Thus, if illusory conjunctions are a correlate of grouping, these studies suggest that orthographic units group letters due to experience.

One concern about the learned-grouping inference arises from Esterman, Prinzmetal, and Robertson (2004). They found that semantic category affects illusory conjunctions: Illusory conjunctions are more likely to occur between a letter or a digit and an O-shaped stimulus if the $\mathrm{O}$ is contextually perceived as the letter $\mathrm{O}$ or the digit zero, respectively. Thus, the unitization of words observed by Prinzmetal and colleagues (Prinzmetal \& Millis-Wright, 1984; Prinzmetal et al., 1986) might not occur at a perceptual level.

Another problem arises from the use of linguistic stimuli, which have the benefit of a lifetime of exposure, a correspondence with vocal stimulation, and associations due to a mixture of co-occurrence frequency and correspondence with Gestalt cues. They are also engineered and uncontrollable, and thus the combinations that produced illusory conjunctions may be confounded with other cues to grouping that determined their selection as linguistic units in the first place. Thus, no causal relationship between training and grouping effects was established by these studies.

These limitations were overcome in the present study. The meaningless nature of our stimuli and the fact that the groupings we induced were completely incidental to the task removed the semantic component from our findings. Our paradigm also allowed a direct demonstration of a causal relationship between group training and transfer.

\section{Visual Statistical Learning}

Finally, our question also closely relates to those posed by researchers who study visual statistical learning (VSL). Fiser and Aslin (2001) found that subjects could recognize shape pairs after short training sessions in which they were exposed to arrays of shapes that contained embedded statistical dependences (e.g., if Shape 1 appeared in the scene, then Shape 2 appeared in the scene, always in the same configuration). These shape pairs were recognized at above-chance levels, even though the training phase was passively viewed. In a similar vein, Brady and Kersten (2003) asked human subjects to observe scenes in which an object was embedded within a field of similarlooking parts, so that the object was camouflaged. Repeatedly viewing such stimuli with the same objects embedded in different camouflaged fields led to significant improvements in both recognition and tracing accuracy. In addition to above-chance recognition of learned associations, subjects can also use the association to facilitate performance in visual search. For example, Chun and Jiang (1999) showed that correlations between distractor and target shapes in visual search could be learned so as to speed up search on trained pairs.

Although VSL can result in the recognition of learned associations, there is no evidence that the trained shapes are actually perceived as a single group. In principle, subjects may be able to recognize shape associates without forming perceptual groups of them, and perceptual groups may form between items without explicit recognition of 
Within-Group Repetition:
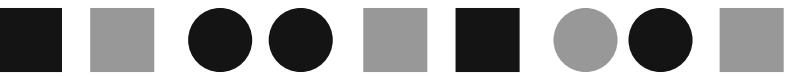

Between-Group Repetition:
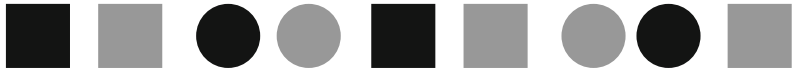

Figure 1. An example of the repetition discrimination task in Palmer and Beck (2007). The task is to find the repetition of color and identify it as gray or black. Repetition discrimination is slower when the color repetition does not coincide with the shape similarity grouping than when it does coincide with the grouping.

cues that lead to grouping. When perceptual grouping occurs (e.g., due to similarity), the resulting group is treated by the visual system as a unit. However, it is hard to claim that grouped units are "recognized."

\section{The Present Study}

In this study, we asked whether visual training can ever lead to perceptual grouping of unconnected, distinct elements. Traditionally, perceptual grouping has been measured by subjective report of grouping (Kubovy \& Wagemans, 1995), which, unfortunately, may be insensitive to short periods of training. As was reviewed above, perceptual grouping is also sometimes measured by asking subjects to recognize the group or to trace the fore- ground object from camouflage (e.g., Brady \& Kersten, 2003), but recognition does not necessarily imply grouping. Palmer and Beck (2007) have recently developed an alternative measure to subjective report, using a paradigm called the repetition discrimination task (RDT; see also Beck \& Palmer, 2002; Vickery, 2008). In this paradigm (Figure 1), subjects view a row of items that are grouped by one dimension (such as shape similarity). The subjects are asked to locate two adjacent items that are the same in another dimension (such as color). Color repetition localization and discrimination are faster when the repeated items belong to a single group than when they cross a group boundary on an irrelevant dimension. This effect is a desirable metric of grouping, because it is impartial and completely unintentional: Exploiting the grouping factor cannot lend a performance advantage to the subjects.

Another measure of grouping, less commonly used, relies on distortions of spatial perception caused by grouping. Coren and Girgus (1980) examined spatial distortions due to various rules of grouping. They asked 94 observers to estimate the distance between two dots. On grouped trials, the dots were grouped together by one of several traditional principles. On ungrouped trials, the two dots belonged to different groups but had the same spacing as on grouped trials. Subjects judged the grouped dots as closer than the ungrouped dots, despite the equivalence of their spacing. Thus, grouping can be assessed by examining the extent to which spatial distortions occur.

In the experiments presented here, we trained subjects on pairings of the stimuli shown in Figure 2 and then tested them in a transfer procedure in which the task was
A

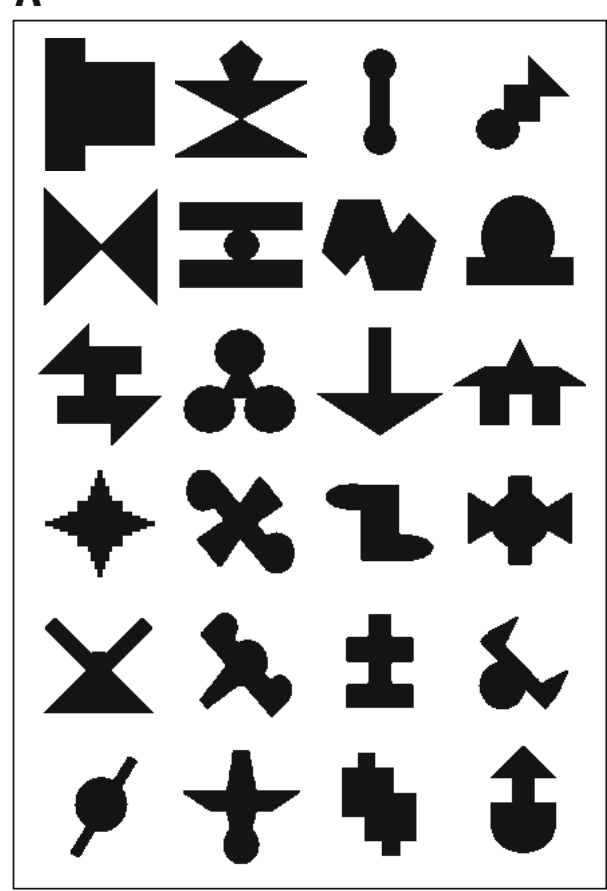

B

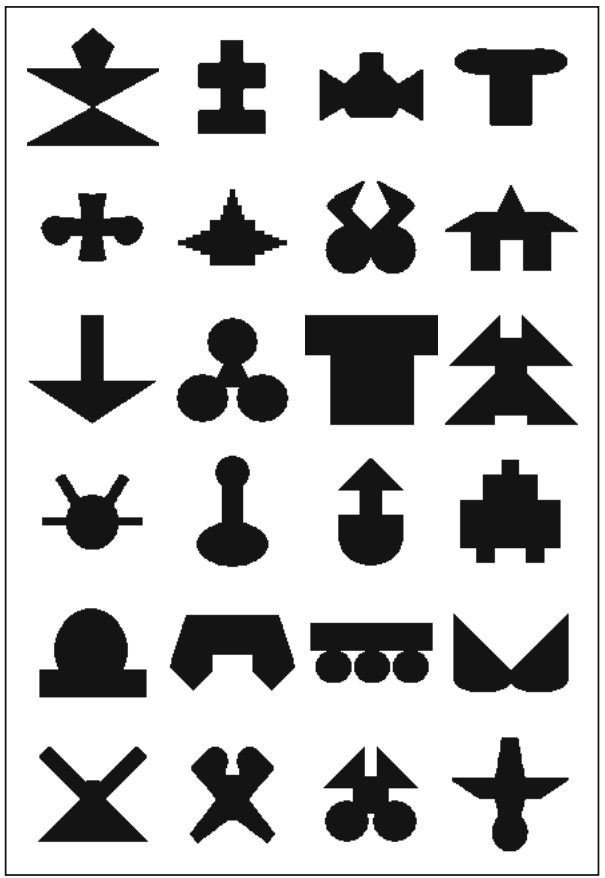

Figure 2. (A) The set of shapes used in all but Experiment 3 (from Fiser \& Aslin, 2001, and TurkBrowne, Jungé, \& Scholl, 2005). (B) Vertically asymmetric shapes used in Experiment 3, composed of some shapes from panel $A$, plus additional ones created for this study. 
to detect a repetition of color (an example trial is shown in Figure $3 \mathrm{~B}$ ) or to adjust the spacing between grouped and ungrouped pairs. If associative learning supports grouping, then in the RDT transfer task, the subjects should be slower to detect a repetition of color if the repetition crosses a learned group boundary than if it occurs within a learned group. Subjects should also show similar distortions of spatial perception due to trained grouping and traditional grouping principles in the distance adjustment task.

In this study, we first identified a case in which associative grouping occurs and then explored some of its properties. Experiment 1 served as an existence proof of this form of learning, in which pairs of shapes became associated by an explicit grouping cue during a training phase, with residual grouping effects in the transfer phase. Experiment 2 confirmed our findings by demonstrating that the trained grouping led to a spatial distortion effect. In Experiment 3, we examined two questions: (1) How durable are these representations? (2) Are these representations flexible to inversions of the original, learned shape pairs? In Experiment 4, we took a closer look at the question of durability, asking whether the dissipation of learning over time is due to decay or interference. Finally, in Experiment 5, we reexamined the flexibility of this learning and asked whether grouping survives when the original learned shapes exchange positions.

\section{EXPERIMENT 1 Transfer of a Grouping Effect Based on Shape Association}

To motivate the training approach used in this study, it is useful to turn to ecological considerations. The binding of unique shapes into groups by association might be dominated, in the real world, by other, more powerful grouping cues - in particular, common fate and connectedness. Ecologically speaking, grouping-by-association might come into play when these cues are ineffective. Thus, in the majority of experience, bottom-up grouping cues dominate scene understanding of groups and units. However, associative grouping will play a role when those cues are invisible due to accidents of viewpoint, lighting, and so on. Taking these factors into consideration, the acquisition of groups might be limited to those situations in which both statistics and visible grouping cues link distinct stimuli together.

In Experiment 1, we tested the hypothesis that associative grouping might be observed when training included visible grouping cues. In the training phase, subjects completed a repetition localization task (Palmer \& Beck, 2007), in which they searched through rows of shapes to find the repetition of color (two items that were side by side and had the same color), with pairs of shapes surrounded by common regions (Palmer, 1992). These cues always surrounded the same shapes. That is, if a row was composed of shapes A, B, C, and D, then whenever these shapes were seen, $\mathrm{A}$ and $\mathrm{B}$ were grouped, and $\mathrm{C}$ and $\mathrm{D}$ were grouped. Afterward, the subjects completed a transfer phase that was similar to the training phase, but with the common region cue removed. If perceptual grouping is insensitive to learning, there should be no difference in reaction times (RTs) for detecting a repetition of color that crosses shapes $\mathrm{BC}$ or DA than when it is contained within $\mathrm{AB}$ or $\mathrm{CD}$. Alternatively, if perceptual groups can form due to statistical association of shapes, RTs for between-groups repetitions of color (over BC or DA) should be longer than those for within-group repetitions $(\mathrm{AB}$ or $\mathrm{CD})$.

\section{Method}

Subjects. Seven individuals completed Experiment 1. The subjects in this and all the other experiments were between the ages of 18 and 35 years and were drawn from the Harvard University community through the Department of Psychology's study pool (except in Experiment 2). The subjects completed approximately $1 \mathrm{~h}$ of testing, for cash payment or course credit.

Stimuli. The shape stimuli were 24 unique shapes adapted from Fiser and Aslin (2001) and Turk-Browne, Jungé, and Scholl (2005) (Figure 2A). They appeared in white or light gray on a medium gray background (illustrated throughout this article as black or gray on a white background, for the sake of depiction). Size varied slightly, but each shape was approximately $1.33^{\circ}$ of visual angle on a side. For each subject, these shapes were randomly allocated to 12 pairings (henceforth, the trained groupings) before the training period began.

Training phase. The training phase of Experiment $1 \mathrm{~A}$ was a variation of the repetition discrimination task described above (Figure $3 \mathrm{~A}$ ). Prior to the training phase, trained groupings were assigned to six configurations. That is, each trained pairing was assigned to another trained pairing. On each trial, a row of 15 evenly spaced shapes appeared, with each item spaced $1.7^{\circ}$ center to center from its neighbors. Each of these shapes was from one of the six configurations, and they were arranged so that the shapes from trained pairs were adjacent. For example, if $\mathrm{AB}$ and $\mathrm{CD}$ were assigned to a configuration, an example trial configuration would be CDABCDABCDA. The starting shape for each trial was chosen at random. Finally, a black rectangular contour $\left(1.4^{\circ} \times 3.2^{\circ}\right)$ surrounded pairs of shapes that were assigned to the same group. For example, black contours would surround AB and CD every time the configuration above was viewed.

The subjects, however, were asked to ignore the shape and the contours and concentrate on color. Color normally alternated from left to right (dark, light, dark, light, . . , with the first color chosen randomly). However, on every trial, two and only two neighbors shared the same color (a dark-dark repetition or a light-light repetition). The subjects' task was to locate this repetition and press the space bar. The items were immediately replaced by black, $\mathrm{X}$-shaped placeholders that masked the stimuli. The subjects were told to position the mouse over the placeholders that were located in the same positions as the repeated items, click the mouse button, and then verify their choice with another keypress (choices could be corrected before the final keypress). They were instructed to do this as quickly as possible without making an error.

The positioning of the color repetition was the key variable. On half the trials, the repetition was within group and was contained within a grouping (e.g., $\mathrm{AB}$ or $\mathrm{CD}$, in our example). On the other half of trials, it was between groups, crossing a grouping boundary (e.g., $\mathrm{BC}$ or DA), as defined by the common region cue.

During the training phase, the subjects completed 240 trials, broken into blocks of 40 trials. There were a total of 120 within-group and 120 between-groups trials, split evenly among the six possible shape pair combinations. Thus, the subjects were exposed to each possible shape pairing exactly 40 times.

Transfer phase. The transfer phase (Figure 3B) was conducted immediately following the training phase and was the same, except for the following differences. First, the common region grouping cues were removed. Second, there were 11 items per row, as compared with 15 during training (in order to reduce RT variance). 
A

Training Phase Examples

Within-Group Trial
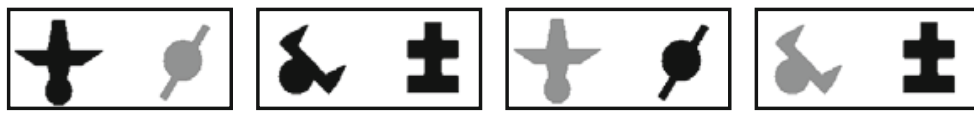

- - - - - - - - - - -

Between-Groups Trial
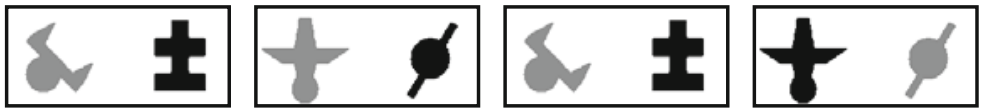

B

Transfer Phase Examples

Within-Group Trial
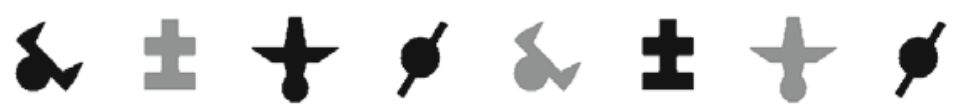
$--$

Between-Groups Trial
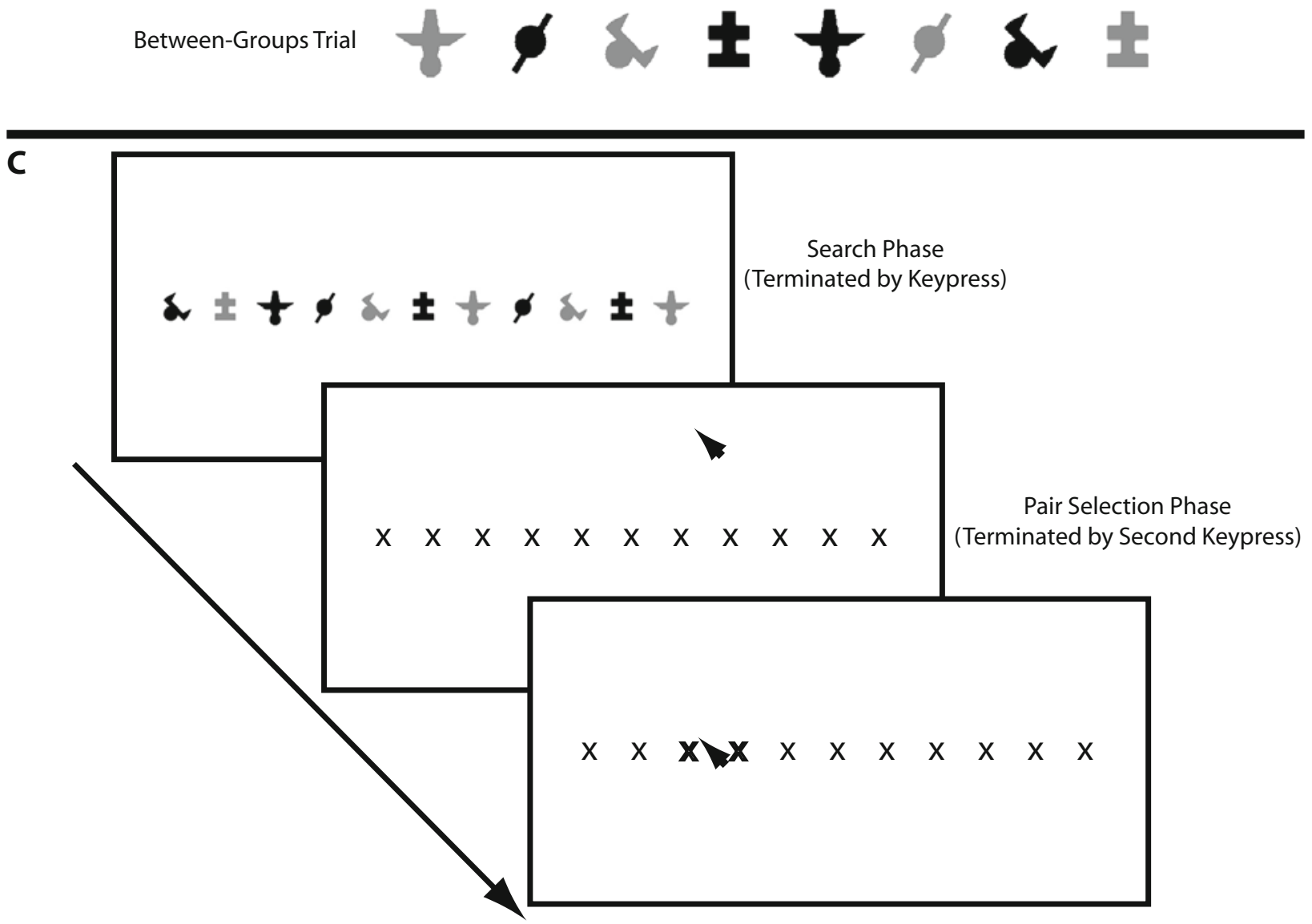

Figure 3. Experiment 1: Examples of training and transfer phase trials in within- and between-groups conditions. (A) In the training condition, shape pairs were consistently grouped by common region. (B) In the transfer condition, the boxes were removed, but the shapes were still consistently paired. In the actual experiment, 15 items were present in training, whereas 11 items were present on each trial of transfer. For simplicity, 8 are shown. The first shape was randomly chosen and could be any of the four shapes that composed the scene. In training, one box had an empty "slot" due to the odd number of targets. (C) A depictive example of a transfer trial for Experiment 1. A different procedure, requiring only an identification response, was used in the later experiments (not shown). 
The subjects completed 4 random practice trials and then 120 trials. Each trial was separated by a $1-\mathrm{sec}$ blank period. Each configuration appeared on 20 trials, composed of 10 within-group and 10 between-groups trials. The trials occurred in a random order and were divided into blocks of 40 trials, between which a short break was permitted. A beep indicated when an error was made. The subjects were told simply that the task was identical, except that the boxes would not be present on the display and fewer items would be present.

The transfer phase of Experiment 1 was designed to probe whether or not the learned pairs of shapes were perceptually grouped due to their statistical association in the training phase. A grouping effect in the training phase was expected (between-groups slower than within-group trials). But would this effect persist when the cues to grouping were removed?

\section{Results}

Training phase. Training phase results were analyzed by binning all trials into between-groups and within-group conditions, on the basis of the location of the color repetition with respect to the trained shape pairs. RTs were analyzed, in this and all the subsequent experiments, by first removing all incorrect trials. Then, RTs that were longer than $10 \mathrm{sec}$ were removed from analysis, to reduce the influence of outliers on the mean. Finally, RTs were averaged separately for each condition and each subject.

For the training phase, an analysis of accuracy showed that the subjects were highly accurate and that accuracy did not differ between the two grouping conditions $(97.9 \%$ in the within-group condition and $97.3 \%$ in the betweengroups condition; $t<1$ ).

The resulting RT analysis (Figure 4) comparing the 120 within-group trials with the 120 between-groups trials showed significant effects of the grouping cue, as was predicted. Average RT was shorter on the within-group trials than on the between-groups trials $[t(6)=4.32, p<$ $.005, d=1.63]$. Thus, our common region grouping cue was effective at producing strong, measurable effects on $\mathrm{RT}$ in the RDT paradigm.

Transfer phase. An RT analysis of the transfer stage was conducted in the same manner as the RT analysis for the training stage, with the exception that a lower cutoff of $7 \mathrm{sec}$ was used in trimming outliers, since the decreased number of items and the removal of the boxes dramatically sped RT overall. ${ }^{1}$

In the transfer phase (Figure 4), between-groups detection was also slowed significantly, relative to the withingroup condition $[t(6)=3.10, p=.02, d=1.17]$. An analysis of accuracy showed that the subjects were, on average, less accurate on between-groups (95.4\%) than on within-group (97.2\%) trials, although this difference was not significant $[t(6)=1.93, p=.10, d=0.73]$, and this accuracy difference was in agreement with the RT grouping effect (i.e., worse for between-groups than for within-group trials).

\section{Discussion}

Experiment 1 provided the first demonstration that a grouping-like effect can be induced by incidental exposure to associated shapes. Interestingly, the introduction of a grouping cue induced a grouping effect even though attention to the shape features of the paired items was neither re-

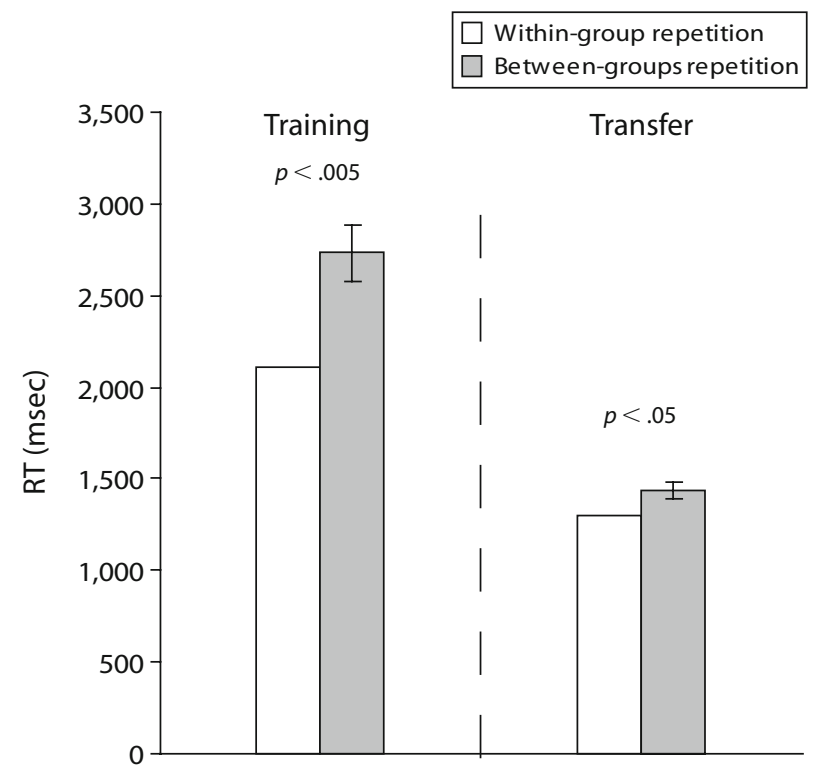

Figure 4. Results of Experiment 1. Bars represent the reaction times (RTs) in between-groups trials and within-group trials in training and transfer phases. The graph on the left depicts the result of the training phase (with boxed items), and the graph on the right depicts the result of the transfer phase (with no box cues to grouping). Error bars represent the standard error of the mean differences between adjacent bars.

quired nor helpful in performing the task. To distinguish this from standard VSL, we term this form of learning associative grouping. We propose that associative grouping binds two objects together after cues that normally group them together are erased and may form an important component of everyday perception. The rest of this study verified and expanded upon some important properties of this effect.

Although the training and transfer phases differed in the number of items and, thus, are not directly comparable, it does appear that the interference effect of the grouping cues was much reduced in the transfer phase. This is not unexpected, since grouping should be viewed as a combination of many factors. The common region cues are a very potent cue to grouping. The association of shapes was due to this cue, and thus, any residue transfer effect would be less than or equal to this effect. On top of this, the presence of such a potent cue likely dominated the effects of shape similarity that randomly occurred between adjacent, ungrouped items. It is not surprising that the association of shapes due to such short training episodes would be less likely to dominate the "natural" grouping factors.

\section{EXPERIMENT 2 \\ Distortions of Spatial Perception Due to Grouping}

Experiment 1 showed that one correlate of perceptual grouping can transfer from a training context in which a grouping cue consistently binds shapes together to a context in which no grouping cue is evident. The association was based on shape identity. However, two caveats remain. First, given that the repetition detection task was 
used in both training and transfer, it is possible that the effect shown in Experiment 1 was task specific, rather than a general one. ${ }^{2}$ Second, the effect shows that a performancebased correlate of grouping can be influenced by trained associations, but is the effect truly "perceptual"? In this experiment, we showed that such training can cause perceptual distortions that are similar to those caused by traditional grouping principles and that these learning effects transfer across very different tasks.

As previously described, Coren and Girgus (1980) found distortions of spatial perception in grouped items, relative to ungrouped items. However, Coren and Girgus's technique required many subjects and showed weak effects that would be expected to be even weaker for grouping based on short, lab-based training episodes. Therefore, we developed a new paradigm inspired by their findings. Our technique involves the adjustment of distance between two items to match the distance between a reference pair. Experiment $2 \mathrm{~A}$ validated this paradigm, using the grouping cue of similarity, and showed a consistent difference between adjustments to a grouped pair with respect to an ungrouped pair and adjustments to an ungrouped pair with respect to a grouped pair. We then employed the same technique in Experiment 2B in the transfer phase, after subjects were trained using the same group training regimen as that used in Experiment 1.

\section{Method}

Subjects. The subjects were 18 to 35 years of age, drawn from paid subject pools at the University of Minnesota and Yale University. Twelve individuals completed Experiment 2A, and 9 completed Experiment 2B.

Stimuli. The same shapes were used in Experiment 2 as in Experiment 1. In Experiment 2A, however, the shapes were either light gray or black, rather than dark gray. In Experiment 2B, they were identical to those in Experiment 1.

Experiment 2A: Task and Procedure. In Experiment 2A, the subjects saw two pairs of shapes on each trial. Examples are shown in Figure 5. The pair on the left was always the reference pair, whereas the pair on the right was always the adjustment pair. The subjects' task was to adjust the center-to-center spacing of the adjustment pair to match the center-to-center spacing of the reference pair. This was accomplished by pressing arrow keys, one of which moved the adjustment pair closer together by 1 pixel $\left(0.034^{\circ}\right)$, whereas the other key moved them apart by 1 pixel. Judgments were unspeeded, and there were no constraints on the number of possible adjustments in a single trial. A final buttonpress signaled that the subjects were satisfied with the adjustment. The items in the reference pair were always separated $1.7^{\circ}$ center to center (50 pixels). The items in the adjustment pair had a random initial separation of between $1.4^{\circ}$ and $2.0^{\circ}$ (41-59 pixels), although the initial separation never precisely matched the reference pair. Pairs were centered vertically and were positioned $15.6^{\circ}$ (400 pixels) apart. However, both the horizontal and vertical positions of each pair were also jittered independently in a $3.9^{\circ}$ (100-pixel) window around these positions on a trial-by-trial basis.

In Experiment 2A, two factors were manipulated. The first was whether the reference pair or the adjustment pair was grouped according to similarity. On grouped-reference trials, the reference pair was grouped by similarity, whereas the adjustment pair was not. On grouped-adjustment trials, the reference pair was not grouped, whereas the adjustment pair was grouped. The second manipulation was of the type of similarity grouping: color or shape. The shapes constituting the reference and adjustment pairs for a given trial were drawn without replacement from the set of shapes used in Experiment 1 . On color-grouping trials, the shapes in the grouped pair were the same color (both black or both light gray), whereas the shapes in the ungrouped pair were different colors (one black and one gray, chosen randomly). All of the items were shaped differently. On shape-grouping trials, the shapes in the grouped pair were identical, whereas the shapes in the ungrouped pair were different (from one another, as well as from the shape in the grouped pair). Their

A

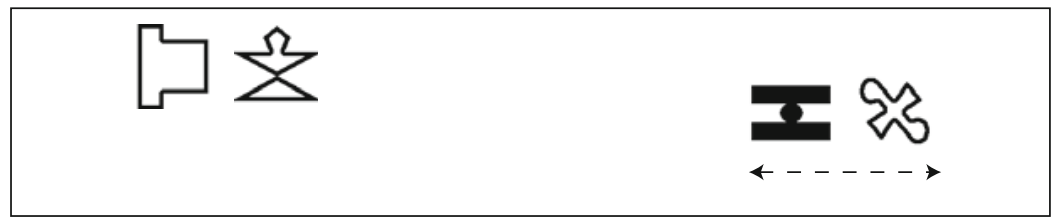

B

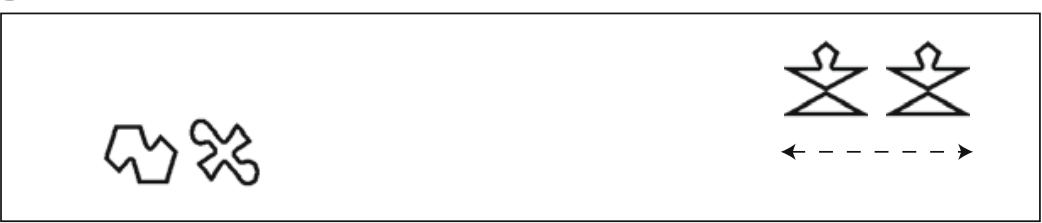

C

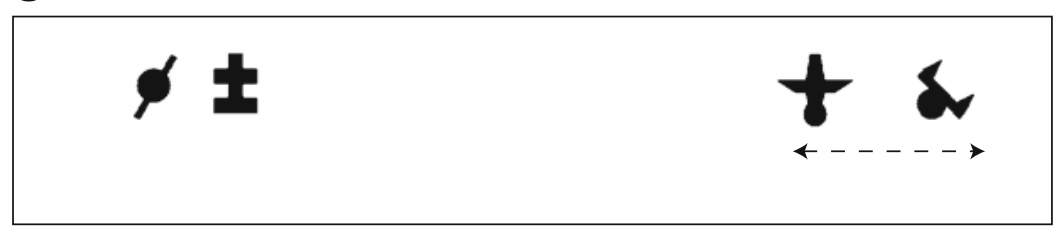

Figure 5. Examples of trials from Experiment 2. Subjects adjusted the distance between shape pairs on the right to match the distances between shape pairs on the left. The arrows were not shown. (A) A color similarity grouped-reference trial. (B) A shape similarity grouped-adjustment trial. (C) An example trial from Experiment 2B. 
colors were all light gray or all black, chosen randomly. The subjects completed 24 trials in each of these four conditions, for a total of 96 randomly intermixed trials.

Experiment 2B: Task and Procedure. Experiment 2B was composed of a training and a transfer stage, like Experiment 1 . The training stage was the same as that in Experiment 1, except that, instead of localizing the repetition and then clicking on placeholders in those positions, the task was to locate the repeated color and respond with one key if the repetition was composed of two dark items and another key if the repetition was composed of two light items. RTs and accuracy were based on these keypresses.

The transfer stage was identical to that in Experiment 2A, except where noted. First, groups were defined by the training stage pairings. Ungrouped pairs were drawn from pairs of shapes that appeared adjacent to one another during training, but separated into different groups by the common region grouping cue. Grouped pairs had appeared within the bounds of the same common region cues during training. For each transfer stage trial, the grouped pair and the ungrouped pair had not appeared in the same configurations during training. Each of the 12 grouped pairs appeared four times during transfer, twice in grouped-reference trials and twice in groupedadjustment trials. Each grouped pair appeared with two different ungrouped pairs, once in each of the two conditions. Thus, a total of 24 grouped-reference and 24 ungrouped-reference trials were completed, in a randomized order.

\section{Results}

Experiment 2A. For each subject, the final spacing of the adjustment was averaged for each trial, separated by grouping condition (grouped-reference or groupedadjustment condition) and type of grouping (color or shape similarity). These values were entered into a $2 \times 2$ repeated measures ANOVA. There was no significant interaction $[F(1,11)=1.8, p=.2]$ and no main effect of grouping type $(F<1)$. However, there was a highly significant effect of reference group assignment $[F(1,11)=$ $33.1, p<.001]$, such that on grouped-reference trials, the subjects adjusted the ungrouped pair to be farther apart than their adjustment of the grouped pairs on groupedadjustment trials. The subjects adjusted pairs to an average of 51.5 pixels apart $(S E M=0.35$; degrees, $M=1.75$, $S E M=0.012)$ on grouped-reference trials, and 50.0 pixels apart $(S E M=0.38$; degrees, $M=1.7, S E M=0.013)$ on grouped-adjustment trials. In both cases, the reference pair was 50 pixels apart. Thus, the subjects were less accurate on grouped-reference than on grouped-adjustment trials and tended to adjust the nongrouped pairs to be farther apart than the reference, grouped pair. The effect was highly reliable for both color and shape similarity $(p<$ .005 for both, post hoc comparisons). These results suggest that perceptual grouping warps the space around grouped elements.

Experiment 2B: Training phase. The results of the training phase were similar to those in Experiment 1. The subjects were much slower to identify the repetition when it crossed a group boundary $(3,313 \mathrm{msec})$ than when it was contained within a group boundary $(2,624 \mathrm{msec})[t(8)=$ $15.6, p<.001]$. There was no corresponding difference between accuracy ( $t<1$; average, 97\% accurate).

Experiment 2B: Transfer phase. In the transfer phase of the experiment, subject adjustment choices based on trained groupings showed a pattern similar to that observed with similarity grouping. On grouped-adjustment trials, the subjects adjusted the grouped pairs to an average distance of 51.0 pixels $(S E M=0.77$; degrees, $M=1.73$, $S E M=0.026)$, as compared with an average adjustment distance of 50.0 pixels ( $S E M=0.73$; degrees, $M=1.70$, $S E M=0.025)$ for ungrouped pairs on grouped-reference trials, a difference that was observed in all the subjects and was significant $[t(8)=3.43, p<.01]$.

\section{Discussion}

Similarity grouping and trained grouping led to similar patterns of spatial distortions in perception. This experiment bolsters support for our claim that the residual repetition discrimination effect observed in Experiment 1 was due to perceptual grouping per se. Two key issues are resolved by this experiment. For one, Experiment 1 left open the possibility that the effect was task specific, since the transfer task matched the training task and, thus, the effect may not have been a general one. In Experiment 2B, a grouping effect transferred between two completely different tasks - a color repetition task and a distance approximation task. Second, the first experiment showed a priming effect, but was it due to distorted perception? Experiment $2 \mathrm{~B}$ suggests that spatial perception is distorted in a similar way for trained groups as it is for grouping by factors such as similarity.

The particular pattern of results observed in Experiment $2 \mathrm{~A}$ is curious. One might expect a different result on the basis of those found by Coren and Girgus (1980). They found that subjects judged the distances between grouped items to be larger than the distances between ungrouped items, all else held equal. Thus, we might expect to see the ungrouped pairs adjusted to be closer together than reality dictated on grouped-reference trials. However, their methods were quite different from ours. Whereas our experiments involved adjusting one pair of items to match another, theirs involved marking a line with an approximate distance. Whereas our experiments involved complex shapes, theirs involved small dots. One possible explanation for our results is that the distortion due to grouping was perceived by the observers, who overcompensated for it by moving the ungrouped pair to be farther apart than the real spacing. Another possibility is that, by asking our subjects to approximately match the center-tocenter distances between two objects, we focused attention on a different aspect of the grouped pairs, such as the internal structure of the bound unit. This might actually be perceived as larger in grouped than in ungrouped pairs of items. A great advantage of our paradigm over Coren and Girgus's technique is that it was highly consistent across observers and produced very strong results with a much smaller sample size. Thus, whatever the final interpretation, this method could prove useful in the future, and it should be investigated further.

\section{EXPERIMENT 3 Flexibility and Durability of Associative Grouping}

Following up on these results, we next probed the flexibility of associative grouping and its durability. In Ex- 
periment 3, we asked how specific this shape association learning was to the original presentation of the shapes and how durable it would be to longer periods of exposure to these shapes following the removal of grouping cues. Specifically, we tested flexibility to inversions of the originally learned shapes, using a new set of 24 shapes (Figure 2B) that were symmetric over the vertical axis and asymmetric over the horizontal axis (including several from the original set that met these qualifications). This manipulation was intended as a probe into the relationship of shape recognition processes to associative grouping. Shape recognition is known to be strongly orientation dependent for shapes that are learned in a canonical orientation (e.g., Jolicœur, 1985). If associative grouping depends on shape recognition processes, inverting the shapes from the learned, canonical orientation would be expected to disrupt recognition and reduce the strength of associative grouping. If, however, associative grouping depends on associations between orientation-independent features of the shapes, the learning effect might be expected to survive inversion.

In our experiment, we assigned one set of subjects, the same-shape group, to the standard training and transfer conditions introduced in Experiment 1. A second set of subjects, the transformed-shape group, received standard training procedures but saw transfer phase displays composed of shapes that were turned upside down, as compared with their training phase presentations. We also doubled the length of the transfer phase in both groups, in order to investigate the durability of the associative grouping effect.

\section{Method}

Other than the noted modifications, all the procedures were identical to those in Experiment 1.

Subjects. The subjects were 38 individuals from the same population as that in Experiment 1, randomly assigned to two separate groups of 19 subjects each.

Stimuli. A new set of 24 shapes was constructed. These shapes were similar to those used in Experiments 1 and 2 (and included some of the same shapes), but their forms were all symmetric about the vertical axis and asymmetric about the horizontal axis (see Figure 2B).

Design. We tested two groups of subjects. For one group, the same-shape group, the training and transfer sessions were identical to those in Experiment 1, except that the new stimulus shapes were employed and the duration of the transfer phase was doubled to 240 trials (split into two 120-trial epochs for analysis). For the second group (the transformed-shape group), the training session was identical to that for the same-shape group, but in the transfer session, the stimuli were inverted (turned upside down).

Half of the subjects in each group were trained with the stimuli oriented as in Figure 2B, whereas the other half were trained with vertically inverted shapes (actually, 10 and 9 subjects received each manipulation, respectively). Thus, any difference between the two groups at transfer could not be due to the shapes' being in one or the other orientation.

\section{Results}

Training phase. Training phase results were consistent with those in Experiment 1, with no differences evident between the two subject groups. Neither group showed a significant effect of grouping condition (within-group compared with between-groups) on accuracy during training (both $p \mathrm{~s}>.3$ ). Both groups showed a significant effect of the grouping factor on RT. For the sameshape group, the average RT on between-groups trials was 3,025 msec, as compared with $2,400 \mathrm{msec}$ on withingroup trials $[t(18)=8.37, p<.001, d=1.92]$. For the transformed-shape group, the average RT on betweengroups trials was $3,110 \mathrm{msec}$, whereas RT on withingroup trials was $2,519 \operatorname{msec}[t(18)=7.85, p<.001$, $d=1.82]$. A mixed-factor ANOVA showed no group $\times$ grouping condition interaction $(F<1)$. Thus, the two groups showed statistically indistinguishable effects of the grouping cues during training.

Transfer phase. Transfer phase results are summarized in Figure 6. A series of planned comparisons on transfer phase accuracy and RT were conducted to address our questions. First, to address the question of flexibility, we examined only the first epoch of the transfer trials. The results for the same-shape group replicated our findings in Experiment 1: A learning effect was clearly observed, with a significant lengthening of RT on between-groups trials, as compared with within-group trials, in both the training and transfer (first epoch) sessions [1,454 vs. 1,526 msec; $t(18)=3.46, p<.003, d=0.79]$. In agreement, accuracy was also slightly, but significantly, worse on the between-groups trials $(97.9 \%)$ than on the within-group trials $(99.0 \%)[t(18)=2.53, p=.02, d=0.58]$. On the other hand, although the transformed-shape group showed significant between-groups versus within-group slowing in the training phase, the transfer session did not reveal any significant learning effect $[1,553 \mathrm{msec}$ in both conditions; $t(18)<1$ ]; there was also no effect on accuracy [98.1\% for within-group vs. $98.0 \%$ for between-groups trials; $t(18)<1]$. Indeed, an ANOVA on RT that included grouping condition as a within-subjects factor and group (same shape or transformed shape) as a between-subjects factor showed a significant interaction $[F(1,36)=4.79$, $\left.p<.05, \eta^{2}=.12\right]$, so that between-groups trials were slower than within-group trials, but only for the sameshape group. This result suggests that the learning is quite specific to the orientation of the shape; simply inverting the shapes eliminates transfer of learning.

How durable are these representations? To answer this question, we compared the first and second epochs of transfer within the same-shape group. In the second epoch of transfer, no between-groups versus within-group effect was observed $[t(18)<1]$; there was a small but insignificant effect on accuracy, so that between-groups trials were slightly less accurate, as before $[t(18)=1.15$, $p=.27, d=0.26]$. An ANOVA on RT revealed that the interaction between the factors of epoch and grouping condition approached significance, so that a grouping effect was observed in the first epoch, but not in the second epoch $\left[F(1,18)=3.80, p=.067, \eta^{2}=.174\right]$. This result suggests that this learning effect is eliminated, either by repeated exposure or by temporal delay, after around 120 trials of transfer, or 20 exposures to each shape pair.

\section{Discussion}

Experiment 3 reveals two interesting properties of associative grouping. First, the grouping measured here 


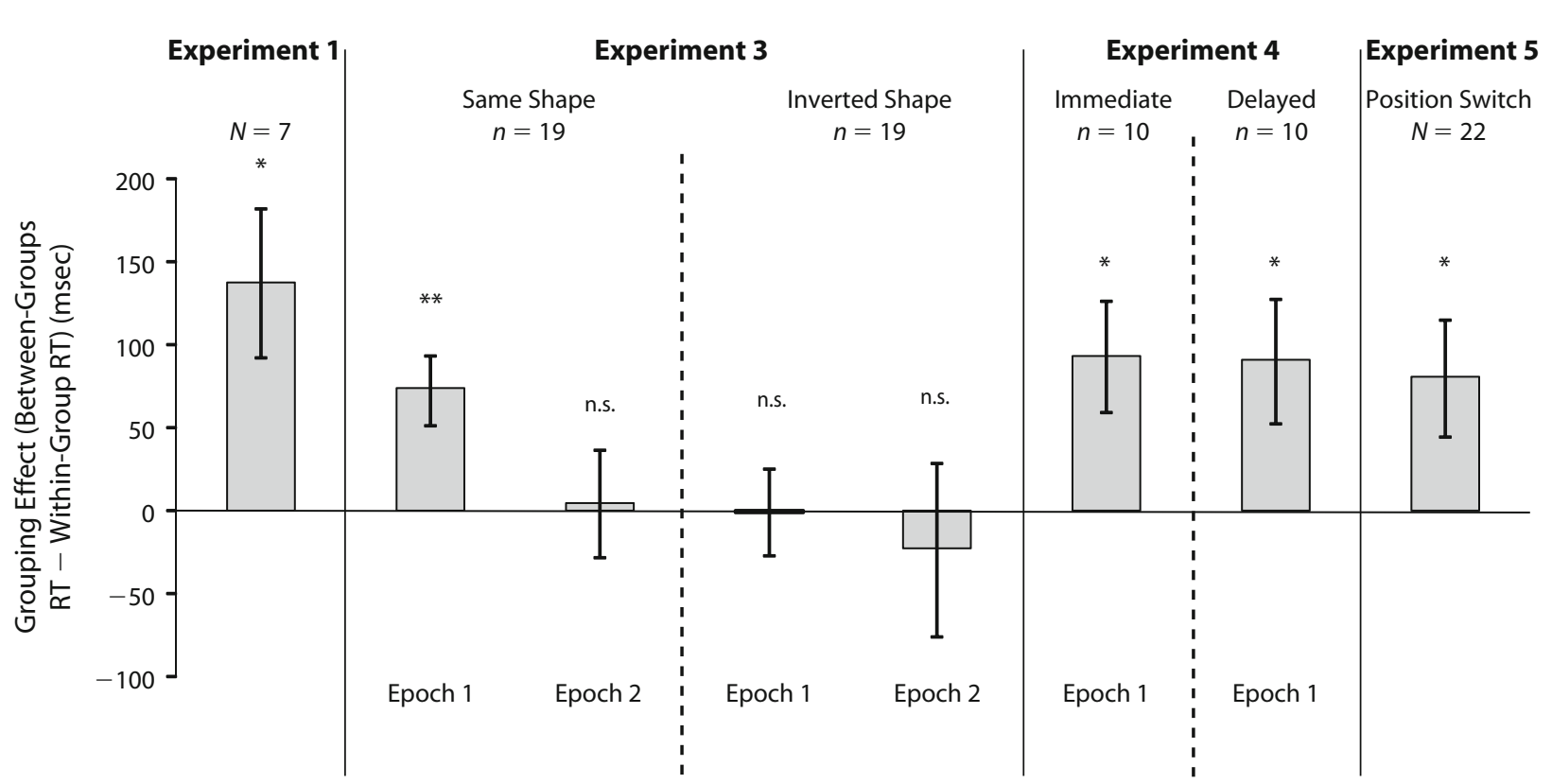

Figure 6. Summary of most important repetition discrimination transfer phase results from Experiments 1 and 3-5. Results plotted are mean reaction time (RT) differences between the between-groups and within-group conditions. A significant positive value implies grouping. Error bars are the standard errors of the difference. Significant results are indicated. $\quad$ * $p<.05 . \quad{ }^{* *} p<.005$.

is specific to the conditions under which learning takes place. This implies that associative grouping is dependent on orientation-specific object recognition to operate. Inverting or otherwise misorienting familiar shapes, such as faces, is widely acknowledged to have a disruptive effect on recognition (e.g., Jolicour, 1985; Yin, 1969). In this experiment, we have shown an interesting twist on an inversion effect, in which interference that normally occurs due to learning is eliminated - an inversion release effect, as contrasted with the inversion interference effects normally discussed in the literature. Experiment 5 delves into the question of flexibility in associative grouping from a slightly different perspective.

It was also found that, with longer transfer phases, the associative grouping effect dissipates. This may pose a challenge to the functional importance of associative grouping. If the dissipation of the effect is due to temporal decay, the removal of the effect after only about 5-6 min of transfer testing would suggest that it is extremely fragile. However, if the effect was due to repeated experience of the shapes in an unpaired context, an ecological argument suggests that associative grouping may still play an important role in vision. As was previously discussed, statistically associated features are typically confounded with grouping cues in the natural world. If the utility of associative grouping is to resolve an ambiguity that occurs relatively rarely, these associations should occur primarily in the (relatively rare) occasions of accidental viewpoints and unusual lighting conditions. If cues to the grouping of items have disappeared, the adaptive system that we are arguing for here should recognize that evidence supporting the grouping of these items has been diminished. In fact, if cues imply instead that the grouping structure was incidental, the adaptive system should abandon its commitment to the learned representation.

Such an adaptive system should show interference from evidence that suggests that groups are no longer relevant (the removal of grouping cues). Experiment 3 demonstrated dissipation of the grouping effect, but the reduction of the effect over time could also be due to simple decay, since the second epoch of transfer was necessarily delayed. In the next experiment, we took up the question of whether the dissipation of associative grouping is due to temporal decay or to repeated exposure to ungrouped shapes, by introducing a fixed delay between training and the first epoch of transfer. If the adaptive system is responsible for the observed dissipation, the delayed group should show the same effect in the first epoch that was observed in a nondelayed group.

\section{EXPERIMENT 4 \\ Dissipation of Associative Grouping: Decay or Interference?}

Experiment 4 was conducted to illuminate the roles of decay and interference in the elimination of the associative grouping effect observed in Experiment 3's second epoch of transfer. The factor of temporal delay between training and the second epoch of transfer and the factor of interference (more experience with ungrouped items between the training and the second epoch) were confounded during Experiment 3. Is the elimination of learning due to this experience, which might be expected to undo the effects of grouping, or was it due to simple temporal decay of the acquired associations? To answer this question, we tested two groups of subjects, the immediate and delayed groups. The immediate group experienced no more than 
normal delay between the training and transfer stages (the minimal time dedicated to setting up the experiment and giving instructions). On the other hand, the delayed group experienced an enforced 10-min delay between training and transfer. This duration was chosen because it was minutes longer than the maximum amount of time that any subject in Experiment 3 spent completing the first epoch of transfer.

\section{Method}

Except where noted, the design and procedure of this experiment were identical to those applied to the same-shape group in Experiment 3.

Subjects. Twenty unique subjects from the same population as that in Experiment 1 completed Experiment 4, with 10 subjects randomly assigned to each of the two groups.

Stimuli. We employed the original 24 shapes used in Experiment 1 in order to ensure that the elimination of the learning was not specific to the newly created shapes.

Task and Procedure. For the immediate group, the procedure was very similar to that for the same-shape group in Experiment 3. However, in this and the subsequent experiments, the repetition discrimination task required pressing a button to indicate whether the repetition was light or dark, just as in the training stage of Experiment 2B.

Other than this modification, the procedure for the immediate group was identical to that for Experiment 3's same-shape condition: 240 training trials were completed, followed as quickly as possible by 240 transfer trials. All trials were binned into 40 -trial blocks.

For the delayed group, the procedure was identical to that for the immediate condition, except that the conclusion of the training session triggered a 10 -min timer. At the end of $10 \mathrm{~min}$, the experimenter set up the transfer phase, and the experiment proceeded as before.

\section{Results}

Training phase. The training phase results replicated those in Experiments 1-3. A mixed-factor ANOVA on RT, with group assignment as a between-subjects factor and grouping condition (within-group vs. between-groups) as a repeated measure factor, showed no interaction and no main effect of grouping (both $F \mathbf{s}<1$ ). There was a strong effect of grouping condition $[F(1,18)=73.8, p<.001$, $d=1.88$ ], such that responses on between-groups trials $(M=3,284 \mathrm{msec})$ were slower than those on within-group trials $(M=2,650 \mathrm{msec})$, as was expected.

Transfer phase. The immediate group's first epoch of transfer was examined to verify that the delay time of $10 \mathrm{~min}$ for the delayed group was appropriate. No subject took longer than $6 \mathrm{~min}$, total, to finish the first epoch of transfer. Average duration of the first transfer stage was $5 \mathrm{~min}, 7 \mathrm{sec}$. Thus, the delayed group began their first epoch of transfer well after the maximum elapsed time between Epoch 1 and Epoch 2.

The results of Experiment 4 (see Figure 6) showed that both the immediate and delayed groups expressed the group learning effect in the first epoch of transfer. The immediate group took, on average, $1,495 \mathrm{msec}$ during within-group trials, whereas they took $1,589 \mathrm{msec}$ on between-groups trials $[t(9)=2.83, p=.02, d=0.57]$. This replicates the results of the previous two experiments. Replicating Experiment 2, the subjects showed a reduced and insignificant effect in the second epoch of transfer $(1,486 \mathrm{msec}$ on within-group trials, 1,511 msec on between-groups trials; $t<1$ ). Thus, the modified task had no influence on the qualitative pattern of results. The delayed group, who completed the experiment after a 10-min delay, also showed significant learning in the first epoch of transfer [1,654 msec vs. $1,746 \mathrm{msec} ; t(9)=$ $2.43, p=.038, d=0.77]$. The results for the delayed group in the second epoch trended toward faster responses on between-groups than on within-group trials $[t(9)=-2.11$, $p=.064, d=-0.67]$. The results for the first epoch of transfer were comparable across the two groups, with no interaction between the within-subjects grouping factor and the between-groups factor of delay $(F<1)$.

\section{Discussion}

The outcome of Experiment 4 proves that decay is not the determining factor of the dissipation of associative grouping, which, instead, probably arises mostly from repeated experience of the objects in an ungrouped formation. Since statistical association is often confounded with grouping cues, associative grouping may play an important role in vision, even if the statistical associations that determine its formation are temporally remote from the time of expression. The mechanism behind associative grouping should be expected to update the probability that two items belong together, even after it has learned to associate two shapes together. If this were not the case, it would rigidly assume that two elements are unitized even with a great deal of experience to the contrary. In these experiments, it is worth noting that persistence over 20 transfer trials is remarkable, given that learning included only 40 exposures under explicit grouping conditions.

\section{EXPERIMENT 5 \\ Flexibility to Transposition of Grouped Shapes}

Experiment 3 demonstrated that associative grouping is not flexible to at least one transformation of the shapes (inversion). This suggests the possibility that this learning might reflect a sort of rigid template formation, in which an exact shape, consisting of the two constituent shapes, or the negative space between the shapes, is acquired. This interpretation does not jibe with the perceptual grouping account that we have presented here, which would intuitively predict more flexibility of transfer than would a simple template theory. However, there is reason to believe that the inversion manipulation would reduce the transfer of learning even under a perceptual grouping account of this phenomenon, given the dependence of shape recognition on orientation. Therefore, we examined further the flexibility of associative grouping in Experiment 5.

In Experiment 5, we exploited the fact that in the prior experiments, the subjects always experienced the shape pairs as occurring in the same order. In other words, a row composed of shapes A, B, C, and D would always present group associates $\mathrm{AB}$ and $\mathrm{CD}$ in that order, and never $\mathrm{BA}$ or DC, during training. We propose that a perceptual grouping account predicts flexibility to transformations of order. A perceptual grouping account would propose that the grouping occurs by virtue of the assignment of token identities to the shapes, and the abstract token identities are associated by the learning process. Therefore, 
the ordering of shapes should be irrelevant as long as the tokens are recoverable. By this account, learning was not exhibited when the shapes were inverted (Experiment 3), due to an impairment of token formation. On the other hand, a strict template view of these results might suggest rigidity to such a transformation. That is, it is possible that what is learned is the joint shape, or joint shape characteristics such as the negative space between the shapes or the joint outline. Thus, the template is activated whenever the two shapes co-occur in a particular formation, and performance is altered on the basis of the activation of this template. By this account, any manipulation that affects the overall joint shape of the pair should reduce the effects of learning, including inverting or transposing the shapes.

In this experiment, we tested subjects under conditions that closely mimicked those of the immediate group in Experiment 4, employing that group as a point of comparison. All the subjects in Experiment 5 were trained as in Experiment 4. In the transfer stage, however, all the groups were reversed in position, so that any trained shape combination $\mathrm{AB}$ was seen in the transfer phase as $\mathrm{BA}$. Although a lack of transfer to such conditions would not rule out the perceptual grouping hypothesis, the existence of transfer would strongly bolster the case for perceptual grouping, as compared with the template hypothesis.

\section{Method}

Subjects. Twenty-two unique subjects from the same population as in Experiment 1 completed Experiment 5.

Design. All the details in Experiment 5 were identical to those for the immediate group in Experiment 4, except for two modifications. First, in the transfer stage, each row's items were reversed in group position. That is, if the training exposed the subjects to rows composed of $\mathrm{A}, \mathrm{B}, \mathrm{C}$, and $\mathrm{D}(\mathrm{ABCDAB} \ldots \mathrm{ABC})$, with $\mathrm{AB}$ and $\mathrm{CD}$ grouped together, the transfer session trials with these shapes reversed the positions of $\mathrm{AB}$ and $\mathrm{CD}$ (e.g., BADCBADCBAD). The second modification was to eliminate the second epoch of transfer in order to shorten the length of the experiment.

\section{Results}

Training phase. Performance during the training phase was similar to that in the prior experiments. There was no significant effect of grouping condition on accuracy ( $98 \%$ in both conditions; $t<1$ ). There was a strong grouping effect on RT, with slower responses on between-groups trials than on within-group trials $[3,628$ vs. $2,870 \mathrm{msec}$; $t(21)=9.09, p<.001, d=1.94]$.

Transfer phase. The results of the transfer phase (see Figure 6) provide convincing evidence for the perceptual grouping hypothesis: The subjects showed a significant grouping effect in the transfer stage, such that responses on within-group trials were faster than those on betweengroups trials, despite the position reversal experienced in the transfer stage [1,653 vs. $1,733 \mathrm{msec} ; t(21)=2.28, p=$ $.03, d=0.49]$. In addition, an accuracy analysis showed a borderline significant result, with slightly less accurate responses on between-groups than on within-group trials [98.9\% vs. $98.0 \% ; t(22)=2.05, p=.053, d=0.44]$.

An interaction test between Experiment 4's immediate group transfer phase results and Experiment 5's transfer phase results showed no significant interaction $(F<1)$, suggesting that the grouping effect was comparable regardless of whether element position was changed or was the same as that in training.

\section{Discussion}

In contrast to the inversion manipulation in Experiment 3 , constituent position reversal resulted in preserved associative grouping. These results are evidence against a strict template hypothesis, demonstrating flexibility in the face of one transformation that would be expected to violate the confines of a template that incorporated both group shapes.

\section{GENERAL DISCUSSION}

These experiments provide further support for the notion that experience alters perceptual grouping, generally, and give the most direct evidence to date that associative learning can induce perceptual grouping. Despite the short duration of training and the irrelevancy of the grouping dimension, exposure to explicitly segmented pairs was sufficient to induce associative grouping effects. This learning effect manifested in longer RTs for locating color repetitions that crossed associated-shape grouping boundaries, as compared with those contained within associated-shape grouping boundaries, implying that it was not imposed artificially by the observers. It also manifested as a spatial distortion effect that matched the spatial distortions observed with grouping by color or shape similarity.

Associative grouping is subject to interference: Repeated observation of the constituent shapes led to an elimination of the associative grouping effect in Experiment 3. However, Experiment 4 demonstrated that this elimination was not due to temporal decay, and we believe that this should not be a source of much doubt concerning the importance of associative grouping in shaping visual processing. The primary role of associative grouping may be to resolve ambiguities that arise from accidents of viewpoint or lighting that leave the structure of a scene in doubt. Although such situations may occur quite often, we suggest that they are rare, as compared with those situations in which a potent grouping cue coincides with statistically associated features. Thus, such associations may be frequently reinforced between episodes of such ambiguity. The elimination of the effect after repeated exposure to ungrouped items reflects the logical operation of such a flexible system.

Associative grouping is inflexible to inversion of individual objects but is flexible to within-group position reversals. In this respect, it also bears a relationship to Peterson and colleagues' findings that shape recognition influences figure-ground segmentation (Peterson \& Gibson, 1994; Peterson et al., 1991); they found that inverting the shapes and, thus, interfering with shape recognition processes also eliminated their effect. This finding also hints that known shape recognition mechanisms, with their sensitivity to orientation (Jolicœur, 1985), may be involved in associative grouping mechanisms, as opposed to a specialized or distinct shape recognition route. 
On the other hand, the flexibility to left-right position reversal observed in Experiment 5 implies that the relationships between constituent features in associative grouping are somewhat abstract and independent of position. This is an important fact, because it reduces the probability that our results were due to visual templates formed by the observer. A strict template formed from, say, repeatedly searching for two objects together should encompass the shapes of both objects in the experienced orientation.

In the introduction, we called attention to the fact that recognition memory is the standard dependent variable in most VSL studies. We claim that recognition memory does not necessarily reflect perceptual grouping per se and that perceptual grouping may not necessarily result in recognition per se. Indeed, in further studies of these effects (Vickery \& Jiang, 2009), we have found that training methods producing extremely high recognition memory rates for shape pairs can lead to no evidence of perceptual grouping (in terms of the speed difference in repetition discrimination). On the other hand, the same basic training method as that used in every experiment in this study leads to a strong repetition discrimination transfer effect but to very poor recognition memory of grouped versus ungrouped pairs. The subjects trained in the procedures used in this study produced recognition rates for grouped versus ungrouped pairs that did not differ significantly from chance. This calls for further study to determine the nature of representations formed from VSL, by employing diverse dependent measures of learning and comparing effects of different kinds of training on these measures.

These results beg a number of other interesting questions. For instance, does the strength of associative grouping depend on the probability that the two shapes are bound together? This question should be asked in future studies of associative grouping. From the present results, it cannot be determined whether associative grouping would ever occur if the constituent shapes appeared grouped with other items, much less whether the strength of the grouping effects would vary depending on the probability that they should be grouped.

Our learning effect bears some commonalities to other priming effects in visual cognition-in particular, the phenomenon of contextual cuing (Chun \& Jiang, 1998). Contextual cuing refers to the speeded visual searches that occur when distractor and target positions recur over the course of many searches, relative to configurations that do not recur. As for contextual cuing, our results may be due to changes in the way that attention is distributed. Also like contextual cuing, associative grouping may be an implicit memory effect: In a separate set of studies, we have found weak or nonexistent recognition memory for associative groups (Vickery \& Jiang, 2009). In contrast to contextual cuing, associative grouping implies perceptual grouping between shapes and occurs despite the fact that it confers no obvious advantage in either the training or the transfer task.

Even Wertheimer (1923) recognized the possibility that learning could be important to grouping. In his famous work on perceptual grouping, Wertheimer pro- posed the "factor of past experience." He argued that experience might shape perceptual grouping, but he failed to produce a compelling demonstration of this that would be similar to those provided for proximity and similarity. In fact, he produced a salient counterexample, placing letters in configurations that led other grouping cues to dominate, which showed that experience could not overcome the other proposed laws of grouping. The view that experience is relatively unimportant to perceptual grouping is finally starting to be challenged. In this study, we have provided further empirical evidence for the factor of experience in perceptual grouping, by demonstrating an associative aspect. We do not contend that this factor has been shown to dominate other grouping factors. However, we propose that the factor of associative experience is to provide guidance to the visual system when other grouping cues fail to produce a strong, unambiguous grouping.

\section{AUTHOR NOTE}

This work was funded by a grant from Harvard University's Ditmar Fund to T.J.V. We thank Joshua Hartshorne, Patrick Cavanagh, and Ken Nakayama for discussions and comments. We also thank Steve Palmer, Mary Peterson, Jeremy Wolfe, and an anonymous reviewer for useful suggestions and comments. Correspondence should be sent to T. J. Vickery, Department of Psychology, Yale University, 2 Hillhouse Ave., New Haven, CT 06520 (e-mail: tim.vickery@gmail.com).

\section{REFERENCES}

Beck, D. M., \& PALMER, S. E. (2002). Top-down influences on perceptual grouping. Journal of Experimental Psychology: Human Perception \& Performance, 28, 1071-1084. doi:10.1037/0096-1523.28.5.1071

Brady, M. J., \& Kersten, D. (2003). Bootstrapped learning of novel objects. Journal of Vision, 3, 413-422. doi:10.1167/3.6.2

ChUN, M. M., \& JIANG, Y. (1998). Contextual cueing: Implicit learning and memory for visual context guides spatial attention. Cognitive Psychology, 36, 28-71. doi:10.1006/cogp.1998.0681

Chun, M. M., \& JiAng, Y. (1999). Top-down attentional guidance based on implicit learning of visual covariation. Psychological Science, 10, 360-365. doi:10.1111/1467-9280.00168

Coren, S., \& Girgus, J. S. (1980). Principles of perceptual organization and spatial distortion: The gestalt illusions. Journal of Experimental Psychology: Human Perception \& Performance, 6, 404-412. doi:10.1037/0096-1523.6.3.404

Dodd, M. D., \& Pratt, J. (2005). Allocating visual attention to grouped objects. European Journal of Cognitive Psychology, 17, 481-497. doi:10.1080/09541440440000122

Esterman, M., Prinzmetal, W., \& Robertson, L. (2004). Categorization influences illusory conjunctions. Psychonomic Bulletin \& Review, 11, 681-686.

FISER, J., \& AsLin, R. N. (2001). Unsupervised statistical learning of higher-order spatial structures from visual scenes. Psychological Science, 12, 499-504. doi:10.1111/1467-9280.00392

Gottschaldt, K. (1926). Über den Einfluss der Erfahrung auf die Wahrnehmung von Figuren. Psychologische Forschung, 8, 1-87.

IVRY, R. B., \& Prinzmetal, W. (1991). Effect of feature similarity on illusory conjunctions. Perception \& Psychophysics, 49, 105-116.

JoLICCEUR, P. (1985). The time to name disoriented natural objects. Memory \& Cognition, 13, 289-303.

KIMCHI, R., \& HADAD, B. S. (2002). Influence of past experience on perceptual grouping. Psychological Science, 13, 41-47. doi:10.1111/1467 $-9280.00407$

Kubovy, M., \& Wagemans, J. (1995). Grouping by proximity and multistability in dot lattices: A quantitative Gestalt theory. Psychological Science, 6, 225-234. doi:10.1111/j.1467-9280.1995.tb00597.x

Marino, A. C., \& SCHOLL, B. J. (2005). The role of closure in defining 
the "objects" of object-based attention. Perception \& Psychophysics, 67, 1140-1149.

MARR, D. (1982). Vision. San Francisco: Freeman.

NeIsser, U. (1967). Cognitive psychology. Englewood Cliffs, NJ: Prentice Hall.

Palmer, S. E. (1992). Common region: A new principle of perceptual grouping. Cognitive Psychology, 24, 436-447. doi:10.1016/0010 -0285(92)90014-S

Palmer, S. E., \& Beck, D. M. (2007). The repetition discrimination task: An objective method for studying perceptual grouping. Perception \& Psychophysics, 69, 68-78.

PALMER, S. E., \& Rock, I. (1994). Rethinking perceptual organization: The role of uniform connectedness. Psychonomic Bulletin \& Review, 1, 29-55.

Peterson, M. A., \& Gibson, B. S. (1994). Must figure-ground organization precede object recognition? An assumption in peril. Psychological Science, 5, 253-259.

Peterson, M. A., Harvey, E. M., \& Weidenbacher, H. J. (1991). Shape-recognition contributions to figure-ground reversal: Which route counts? Journal of Experimental Psychology: Human Perception \& Performance, 17, 1075-1089. doi:10.1111/j.1467-9280.1994 .tb00622.x

Prinzmetal, W., \& Keysar, B. (1989). A functional theory of illusory conjunctions and neon colors. Journal of Experimental Psychology: General, 118, 165-190. doi:10.1037/0096-3445.118.2.165

Prinzmetal, W., \& Millis-Wright, M. (1984). Cognitive and linguistic factors affect visual feature integration. Cognitive Psychology, 16, 305-340. doi:10.1016/0010-0285(84)90012-4

Prinzmetal, W., Treiman, R., \& Rho, S. H. (1986). How to see a reading unit. Journal of Memory \& Language, 25, 461-475. doi:10.1016/0749-596X(86)90038-0

RoelfSema, P. R. (2006). Cortical algorithms for perceptual grouping. Annual Review of Neuroscience, 29, 203-227. doi:10.1146/annurev .neuro.29.051605.112939

Treisman, A. (1982). Perceptual grouping and attention in visual search for features and for objects. Journal of Experimental Psychology: Human Perception \& Performance, 8, 194-214. doi:10.1037/0096 -1523.8.2.194
Treisman, A., \& SchmidT, H. (1982). Illusory conjunctions in the perception of objects. Cognitive Psychology, 14, 107-141. doi:10.1016/0010 $-0285(82) 90006-8$

Turk-Browne, N. B., Jungé, J. A., \& Scholl, B. J. (2005). The automaticity of visual statistical learning. Journal of Experimental Psychology: General, 134, 552-564. doi:10.1037/0096-3445.134.4.552

Ullman, S. (2007). Object recognition and segmentation by a fragment-based hierarchy. Trends in Cognitive Sciences, 11, 58-64. doi:10.1016/j.tics.2006.11.009

ViCKERY, T. J. (2008). Induced perceptual grouping. Psychological Science, 19, 693-701. doi:10.1111/j.1467-9280.2008.02144.x

VICKERY, T. J., \& JiANG, Y. V. (2009). How associative groups are and are not formed. Manuscript in preparation.

Wertheimer, M. (1923). Untersuchungen zur Lehre von der Gestalt. II. Psychologische Forschung, 4, 301-350. [Partial translation in W. D. Ellis (Ed.) (1950). A sourcebook of Gestalt psychology (pp. 71-81). New York: Humanities Press]

YIN, R. K. (1969). Looking at upside-down faces. Journal of Experimental Psychology, 81, 141-145. doi:10.1037/h0027474

Zemel, R. S., Behrmann, M., Mozer, M. C., \& Bavelier, D. (2002). Experience-dependent perceptual grouping and object-based attention. Journal of Experimental Psychology: Human Perception \& Performance, 28, 202-217. doi:10.1037/0096-1523.28.1.202

\section{NOTES}

1. RT cutoffs used throughout (10 sec for training, $7 \mathrm{sec}$ for transfer) were arbitrary and were meant to remove unreasonably long RTs, on the basis of the range of RTs observed in pilot data. In Experiment 1, the 7 -sec trimming procedure did not eliminate any trials. Trimming by a different method (by eliminating RTs that were more than three standard deviations from the cell mean for each subject) did not significantly alter the results observed in these experiments.

2. We thank Mary Peterson for raising this question.

(Manuscript received May 26, 2008; revision accepted for publication December 20, 2008.) 